\title{
Size-Dependent Plasmonic Resonances from Large-Scale Quantum Simulations
}

\author{
Hongping Xiang, ${ }^{\dagger} \mathrm{Xu}$ Zhang, ${ }^{\dagger}$ Daniel Neuhauser, ${ }^{\dagger}$ and Gang $\mathrm{Lu}^{*}{ }^{\dagger}$ \\ ${ }^{\dagger}$ Department of Physics and Astronomy, California State University Northridge, Northridge, California 91330-8268, United States \\ ${ }^{\ddagger}$ Department of Chemistry and Biochemistry, University of California, Los Angeles, California 90095-1569, United States
}

\section{Supporting Information}

ABSTRACT: For metallic nanoparticles less than $10 \mathrm{~nm}$ in diameter, localized surface plasmon resonances (LSPRs) become sensitive to the quantum nature of conduction electrons. In this regime, experimental probes of size-dependent LSPRs are particularly challenging, and contradictory results are often reported. Unfortunately, quantum mechanical simulations based on time-dependent Kohn-Sham density functional theory (TD-KSDFT) are computationally too expensive to tackle metal particles larger than 2 $\mathrm{nm}$. Herein, we present a time-dependent orbital-free density functional theory (TDOFDFT) that accurately captures the dynamic response of electrons in the presence of realistic ionic potentials. The TD-OFDFT method offers a comparable accuracy as TDKSDFT but with a much lower computational cost. Using TD-OFDFT, we study sizedependent LSPRs on $\mathrm{Na}$ nanoparticles with diameters from 0.7 to $12.3 \mathrm{~nm}$. The optical absorption spectra exhibit a nonmonotonic behavior from blue shift to red shift and back to blue shift as the particle size decreases. Three principal plasmon modes are identified, and their physical origins are elucidated. Competing physical mechanisms responsible

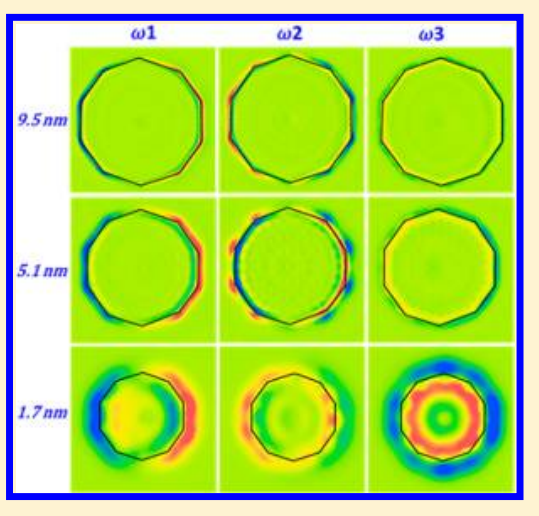
for the nonmonotonic size dependence are discussed. The TD-OFDFT provides a unified theoretical framework that bridges the gap between classical electromagnetic theory and quantum mechanical theory for plasmonics and nanophotonics.

SECTION: Plasmonics, Optical Materials, and Hard Matter

$\mathrm{M}$ etallic nanoparticles are attracting much attention recently owing to their unique physical and chemical properties that are beyond their bulk counterparts and can be tuned based upon their size, shape, composition, and environment. ${ }^{1-5}$ In particular, the collective electron oscillations on metal surfaces, known as local surface plasmon resonances (LSPRs), enable strong optical absorption and scattering in the subwavelength scale, leading to novel applications in chemical and biological sensing, imaging, optoelectronics, energy harvesting and conversion, and medicine. ${ }^{6-11}$ However, in order to advance such applications, one needs to control LSPRs in metal nanostructures. It turns out that the control of nanoparticle size represents one of the most effective approaches to harness the tremendous potentials of plasmonics and nanophotonics. Crucial to this endeavor is the size dependence of LSPRs in nanostructures, which unfortunately remains poorly understood due to the quantum nature of the phenomena, especially for particle sizes from 2 to $20 \mathrm{~nm}$. Therefore, it is scientifically and technologically important to understand, predict, and ultimately control plasmonic responses in nanostructures.

Experimentally, there have been controversies on the size dependence of LSPRs in metallic nanostructures. For example, Parks et al. reported that the optical spectra of small Na clusters exhibited a red shift from 3.4 to $2.4 \mathrm{eV}$ as the cluster size decreased from 300 to 10 atoms. ${ }^{12}$ Reiners et al. examined the optical response of spherical $\mathrm{Na}_{j}{ }^{+}$particles $(9 \leq j \leq 93)$ and observed a red shift as the particle size decreased from 93 to 41 atoms followed by a blue shift as the particles shrank from 41 atoms to 9 atoms, ${ }^{13}$ contradictory to the results of Parks et al. Using photodepletion spectroscopy, Kappes et al. studied the optical absorption of small $\mathrm{Na}$ clusters ( $<20$ atoms) and found an irregular size dependence. ${ }^{14}$ To the best of our knowledge, there is no experimental report on the size dependence of LSPRs for larger Na nanoparticles with more than 300 atoms. However, there are experimental results on Ag nanoparticles, which are also controversial. For instance, monodispersed Ag nanoparticles stabilized with organic ligands exhibited a blue shift as the particle diameter was $d<20 \mathrm{~nm}$ and then a strong red shift as $d<12 \mathrm{~nm} .{ }^{15}$ Scholl et al. examined plasmon resonances of individual ligand-free and substrate-free $\mathrm{Ag}$ nanoparticles and found a blue shift as the particle size was between 2 and $20 \mathrm{~nm} .^{16}$ Therefore, theoretical investigations are imperative to resolve the experimental controversies and more importantly to provide fundamental understanding of the size-dependent LSPRs.

However, theoretical investigation of plasmonic resonances is equally challenging for particle sizes between 2 and $20 \mathrm{~nm}$. This length scale is too small for classical electromagnetic theories to be valid but too large for full-fledged quantum simulations to be

Received: January 30, 2014

Accepted: March 18, 2014

Published: March 18, 2014 
feasible. Classical theories often fail to capture quantum resonances, producing results contradictory to experiments for particle sizes less than $10 \mathrm{~nm} .{ }^{16-18}$ Quantum mechanical simulations based on the time-dependent Kohn-Sham density functional theory (TD-KSDFT) are, in principle, capable of describing quantum resonances. For example, Nordlander's group has examined the plasmonic resonances of nanoparticle dimers, ${ }^{19,20}$ nanowires, and nanowire dimers ${ }^{21,22}$ using TDKSDFT. However, such calculations are computationally demanding and are currently limited to particles smaller than $2 \mathrm{~nm}$ (100 electrons) with pseudopotentials. ${ }^{23}$ Even when the jellium model is used, TD-KSDFT can treat no more than a few thousand electrons ( 2000 electrons) routinely. ${ }^{19-22}$ The only exception to this general rule is the work by Prodan et al. for metallic nanoshells, which assumed spherical symmetry of the wave functions in conjunction with the jellium model. ${ }^{24}$ Therefore, it is crucial to develop an accurate and expeditious quantum mechanical method that can provide a unified theoretical framework to predict and understand LSPRs in metallic nanostructures.

In this Letter, we introduce a time-dependent orbital-free density functional theory (TD-OFDFT) that retains the accuracy of TD-KSDFT at a fraction of its computational cost. Using TD-OFDFT, one can treat particle sizes up to 20 $\mathrm{nm}$, thus bridging the quantum and classical plasmonics. Not only does TD-OFDFT yield excellent agreements with both TD-KSDFT and experimental results for smaller nanoparticles, it also reproduces experimental observations for larger nanoparticles that are beyond the reach of TD-KSDFT. The success of TD-OFDFT is due to the incorporation of two important elements that are missing in previous theoretical efforts. ${ }^{25}$ The first is introduction of a dynamic kinetic energy potential (DKEP) ${ }^{26}$ that ensures the correct frequencydependent and wave-vector-dependent linear response of electron gas; the second is the use of ab initio pseudopotentials ${ }^{27}$ for describing the positive charge background beyond the jellium model.

In this Letter, we first use smaller $\mathrm{Na}$ nanoparticles as examples to demonstrate the accuracy of the TD-OFDFT method by comparing its results to those of TD-KSDFT and experiments. We then apply the method to examine sizedependent plasmonic resonances of individual ligand-free $\mathrm{Na}$ nanoparticles in the quantum size regime $(12.3>d>0.7 \mathrm{~nm})$. We find that the optical absorption spectra of $\mathrm{Na}$ nanoparticles exhibit an intriguing nonmonotonic behavior (blue shift-red shift-blue shift) as the size decreases. An analysis on the electronic charge density indicates the coexistence of three principal resonance modes. The competing physical mechanisms responsible for the observed nonmonotonic size dependence are illustrated. This work resolves the longstanding controversies on the size-dependent LSPRs in metallic nanoparticles and provides a first-principles theoretical framework to understand, predict, and ultimately control plasmonic responses in nanostructures.

Instead of treating many orbitals as in TD-KSDFT, the TDOFDFT approach is based on a single orbital $\Psi$, which is related to the time-dependent charge density $\rho(\vec{r}, t)$ via

$$
\Psi(\vec{r}, t)=\sqrt{\rho(\vec{r}, t)} \mathrm{e}^{\mathrm{i} \chi(\vec{r}, t)}
$$

and is normalized to the total number of electrons; $\chi$ is a phase factor. Note that, arguably, this method may be better labeled as a "time-dependent single-orbital", but we will use the prevailing notation of TD-OFDFT in this Letter. $\Psi$ is determined by solving a Schrodinger-like equation

$$
\begin{aligned}
\mathrm{i} \frac{\partial \Psi}{\partial t}= & -\frac{\Delta \Psi}{2}+\left(\nu_{\mathrm{DKEP}}[\rho](\vec{r}, t)+\int \frac{\rho(\vec{r}, t)}{\left|\vec{r}-\vec{r}^{\prime}\right|} \mathrm{d} \vec{r}^{\prime}\right. \\
& \left.+V_{\mathrm{ion}}(\vec{R})+\nu_{\mathrm{XC}}[\rho](\vec{r}, t)\right) \Psi
\end{aligned}
$$

where the symbols are defined according to the usual conventions. Here, a local pseudopotential is used for $V_{\text {ion }}(\vec{R})$ instead of a jellium background. The atomic-centered local pseudopotential is constructed by inverting the KohnSham equations on the bulk valence electron density using a modified ABINIT code ${ }^{27,28}$ (see the Supporting Information). $\nu_{\mathrm{DKEP}}$ in eq 2 is the DKEP, including three contributions, as shown in eq 3

$$
\nu_{\mathrm{DKEP}}=\frac{\left(3 \pi^{2}\right)^{2 / 3}}{2} \rho^{2 / 3}-(a-1) \frac{\Delta|\Psi|}{2|\Psi|}+\nu_{\mathrm{ker}}
$$

They are the Thomas-Fermi contribution (exact for a uniform electron gas), von Weizsacher contribution (exact for one electron), and a dynamic kernel term $\nu_{\text {ker }}$. Here, $a$ is the numerical coefficient of the von Weizsacher term. The dynamic kernel term is further decomposed into a static nonlocal kinetic energy potential $\nu_{\text {ker }}^{\mathrm{s}}$ as well as a dynamic term $\nu_{\text {ker }}^{\mathrm{d}}$

$$
\nu_{\mathrm{ker}}=\nu_{\mathrm{ker}}^{\mathrm{s}}+\nu_{\mathrm{ker}}^{\mathrm{d}}
$$

Here, $\nu_{\text {ker }}^{\text {s }}$ represents the modern kernel term ${ }^{29}$ for the static OFDFT potential, which depends on the instantaneous electron density $\rho(\vec{r}, t)$ via $\nu_{\mathrm{ker}}^{\mathrm{s}}=\delta V_{\mathrm{s}} / \delta \rho(\vec{r}, t)$. Typically, $V_{\mathrm{s}}$ takes the following form

$$
\begin{aligned}
V_{s}= & \int \rho\left(\vec{r}^{\prime}, t\right)^{\alpha} K\left(\rho\left(\vec{r}^{\prime}, t\right), \rho\left(\vec{r}^{\prime \prime}, t\right),\left|\vec{r}^{\prime}-\vec{r}^{\prime \prime}\right|\right) \\
& \times \rho\left(\vec{r}^{\prime \prime}, t\right)^{\beta} \mathrm{d} \vec{r}^{\prime} \mathrm{d} \vec{r}^{\prime \prime}
\end{aligned}
$$

where $K$ is a material-specific density-dependent kinetic energy kernel.

The crucial ingredient of the formalism is the $\nu_{\mathrm{ker}}^{\mathrm{d}}$ term in eq 4 , introduced to ensure the proper frequency-dependent linear response or the correct dynamic susceptibility of the electron gas. ${ }^{26}$ It manifestly vanishes for the static response and is written as

$$
\nu_{\mathrm{ker}}^{\mathrm{d}}(\vec{r}, t)=\frac{\mathrm{d} y(\vec{r}, t)}{\mathrm{d} t}
$$

and

$$
\begin{aligned}
y(\vec{r}, t)= & \sum_{j} d_{j} \rho(\vec{r}, t)^{\alpha_{j}} \int \mathrm{e}^{-\kappa_{j}\left|\vec{r}-\vec{r}^{\prime}\right|-\mathrm{i} \omega_{j}\left(t-t^{\prime}\right)} \\
& \times \rho\left(\vec{r}^{\prime}, t^{\prime}\right)^{\beta_{j}} \mathrm{~d} \vec{r}^{\prime} \mathrm{d} t^{\prime}+\text { c.c. }
\end{aligned}
$$

The ansatz for $y(\vec{r}, t)$ takes cue from the static kinetic energy potential; $d_{j}, \kappa_{j}$, and $\omega_{j}$ are complex parameters, and for simplicity, the exponentials $\alpha_{j}$ and $\beta_{j}$ are taken to be real, as in the static case. On the basis of the TD-OFDFT formalism, one can derive the dynamic (wave-vector- and frequency-dependent) susceptibility, $\chi_{0}\left(q, \omega, \rho_{0}\right)$, for an initial charge density $\rho_{0}{ }^{26}$ These parameters are then obtained by fitting the TD-OFDFT dynamic susceptibility $\chi_{0}\left(q, \omega, \rho_{0}\right)$ to the exact dynamic susceptibility of a homogeneous electron gas with the same density of $\rho_{0}$ (see the Supporting Information). Note that the 
theoretical formalism and numerical fitting procedure are general and can be applied to alkali and even noble metals. Recently, an angular-momentum-dependent static OFDFT method has been developed, ${ }^{30}$ which renders OFDFT applicable to metals with localized d electrons. Therefore, it is feasible to incorporate both the relativistic effect (via angular momentum pseudopotentials) and contributions from $d$ electrons in the present formalism. Because the relevant revisions are entirely on the static contributions, we do not anticipate technical difficulties to implement the revisions. As we previously fitted the parameters for sodium, ${ }^{26}$ we use $\mathrm{Na}$ particles as examples to explore size-dependent LSPRs in this Letter. Because TD-OFDFT deals with electron density as opposed to wave functions, the computational cost is about the same between, say, $\mathrm{Na}$ and $\mathrm{Ag}$ clusters. The TD-OFDFT approach is implemented in real space using conventional exchange-correlation functionals.

First, we demonstrate the accuracy of the TD-OFDFT method by comparing its predictions to those from TDKSDFT and experiments for small particles. In particular, four positively charged nanoparticles, $\mathrm{Na}_{5}{ }^{+}, \mathrm{Na}_{7}{ }^{+}, \mathrm{Na}_{9}{ }^{+}$, and $\mathrm{Na}_{55}{ }^{+}$, are studied using both TD-OFDFT and TD-KSDFT methods. In all cases, we find excellent agreements between the two methods in terms of plasmon energy-the quantity of interest in plasmonics. Here, we only present the results of $\mathrm{Na}_{9}{ }^{+}$, while the results of $\mathrm{Na}_{5}^{+}, \mathrm{Na}_{7}^{+}$, and $\mathrm{Na}_{55}{ }^{+}$are discussed in the Supporting Information. We use a tricapped trigonal prism $\left(D_{3 h}\right)$ geometry to model a $\mathrm{Na}_{9}{ }^{+}$particle, as shown schematically in the inset of Figure 1. This structure is the lowest-energy

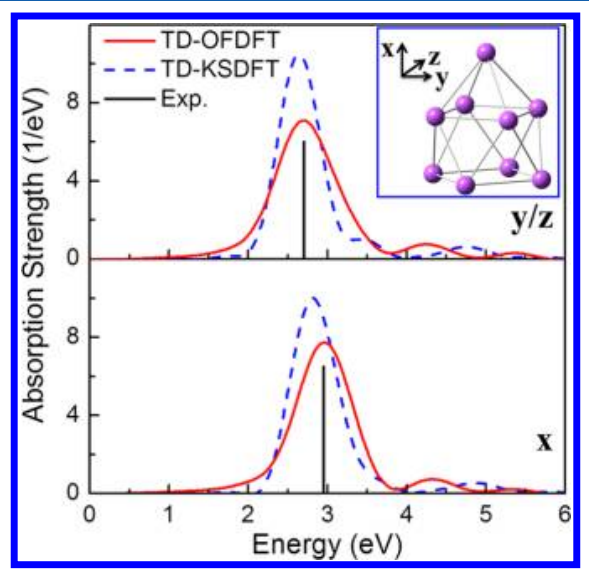

Figure 1. The optical absorption spectra of the $\mathrm{Na}_{9}{ }^{+}$particle along the $x, y$, and $z$ axes from the TD-OFDFT and TD-KSDFT calculations. The atomic structure of the particle is shown in the inset. The black vertical lines indicate the experimental values.

configuration for $\mathrm{Na}_{9}{ }^{+31}$ We calculate the longitudinal optical adsorption spectra using both TD-KSDFT and TD-OFDFT along three polarization directions. As displayed in Figure 1, we find one absorption peak in each polarization direction; owing to the symmetry, the adsorption spectra in $y$ and $z$ are identical. The area under the adsorption curve represents the oscillator strength. The plasmon energy from TD-OFDFT is 2.70 and $2.96 \mathrm{eV}$, respectively, along the $x$ and $y$ (or $z$ ) directions. The corresponding energy from TD-KSDFT is 2.64 and $2.82 \mathrm{eV}$, both in excellent agreement with the TD-OFDFT values (less than $5 \%$ error). Although the atomic structure of the $\mathrm{Na}_{9}{ }^{+}$ particle and the polarization direction were unknown, two adsorption peaks were observed $(2.70$ and $2.95 \mathrm{eV})$ in experiment; ${ }^{32}$ these energies matched exceedingly well the TD-OFDFT results. Hence, we establish that the TD-OFDFT method provides an accurate description of the plasmon resonances for small $\mathrm{Na}$ nanoparticles. Additionally, in TDOFDFT, the computational cost scales linearly with the system size, whereas in TD-KSDFT, the scaling is cubic. Therefore, TD-OFDFT is computationally much more efficient than TDKSDFT, particularly for larger particles.

Using TD-OFDFT, we examined the LSPRs of $\mathrm{Na}$ nanoparticles in the quantum size regime with $d=0.7-12.3$ $\mathrm{nm}$; the largest particle contains over 17800 atoms. The nanoparticles are in a symmetric $I_{h}$ icosahedral shape, which was reported to be experimentally stable. ${ }^{15}$ The highly symmetric structure renders the analysis of plasmonic resonances more convenient without the complication of anisotropy. Because particle shape influences optical spectra considerably, $^{33}$ we examine the particles with the same icosahedral shape to explore exclusively the size dependence of LSPRs. In Figure 2, we display the optical absorption spectra of $13 \mathrm{Na}$ particles (left panel) and the peak value of the optical absorption spectra as a function of the diameter (right panel). The calculated absorption energies are between 2.92 and 3.12 $\mathrm{eV}$ for the nanoparticles examined here, which agree well with previous TD-KSDFT results ${ }^{34}$ ranging from 2.7 to $3.0 \mathrm{eV}$ for particle sizes between 15 to 331 atoms. The energy differences are mainly due to different shapes of the clusters (the icosahedral shape versus bcc) used in the calculations. The most distinctive feature of the results is nonmonotonic size dependence of LSPRs. The plasmon energy is blue-shifted as the particle size decreases from 12.3 to $8.8 \mathrm{~nm}$. As the particle size is further reduced, a strong red shift takes over, with the lowest absorption energy at $d=2.9 \mathrm{~nm}$. With continual shrinking of the particles, the energy is blue-shifted again. Thus, we can classify the size dependence of LSPRs into three regions, (I) blue shift $(d>8.8 \mathrm{~nm})$, (II) red shift $(2.9<d<8.8$ $\mathrm{nm})$, and (III) blue shift $(d<2.9 \mathrm{~nm})$.

In order to understand this nonmonotonic size dependence, we analyze the optical absorption of three selected nanoparticles in more details. In Figure 3a, we display the optical absorption spectra of $\mathrm{Na}$ nanoparticles with $d=9.5\left(\mathrm{Na}_{8217}{ }^{+}\right)$, $5.1\left(\mathrm{Na}_{1415}{ }^{+}\right)$, and $1.7 \mathrm{~nm}\left(\mathrm{Na}_{55}{ }^{+}\right)$, exemplifying, respectively, regions I, II, and III. Shown in Figure 3a, in addition to the strongest absorption peak at $2.97 \mathrm{eV}$, there are two weaker peaks at around 4.0 and $5.0 \mathrm{eV}$ for $\mathrm{Na}_{55}{ }^{+}$. This multiple-peak feature of $\mathrm{Na}_{55}{ }^{+}$along with the asymmetric adsorption spectra for the two other particles hints that multiple resonance modes may be present. To verify this speculation, we resolve each absorption spectrum as a sum of Gaussian functions ${ }^{35}$ and derive three distinctive resonance modes termed $\omega 1, \omega 2$, and $\omega 3$ in Figure 3a (green curves). They correspond to 2.97, 3.96, and $4.78 \mathrm{eV}$ for $\mathrm{Na}_{55}{ }^{+} ; 3.02,3.35$, and $4.45 \mathrm{eV}$ for $\mathrm{Na}_{1415}{ }^{+}$; and $3.04,3.48$, and $4.65 \mathrm{eV}$ for $\mathrm{Na}_{8217}{ }^{+}$, respectively. Indeed, the sum of the three Gaussians (red curve) matches very well the original absorption spectrum (black curve) for each particle, with the only exception at the low-energy range of $\mathrm{Na}_{55}{ }^{+}$. Therefore, one can try to understand the size dependence of LSPRs in terms of these resonance modes.

In Figure 3b, we present the induced charge density (relative to the equilibrium values) evaluated at the three frequencies, $\omega 1, \omega 2$, and $\omega 3$. The charge density was obtained by Fourier transform of the charge density distribution from the time domain to the frequency domain. For each particle, the induced charge density at $\omega 1$ exhibits a typical dipolar plasmon 


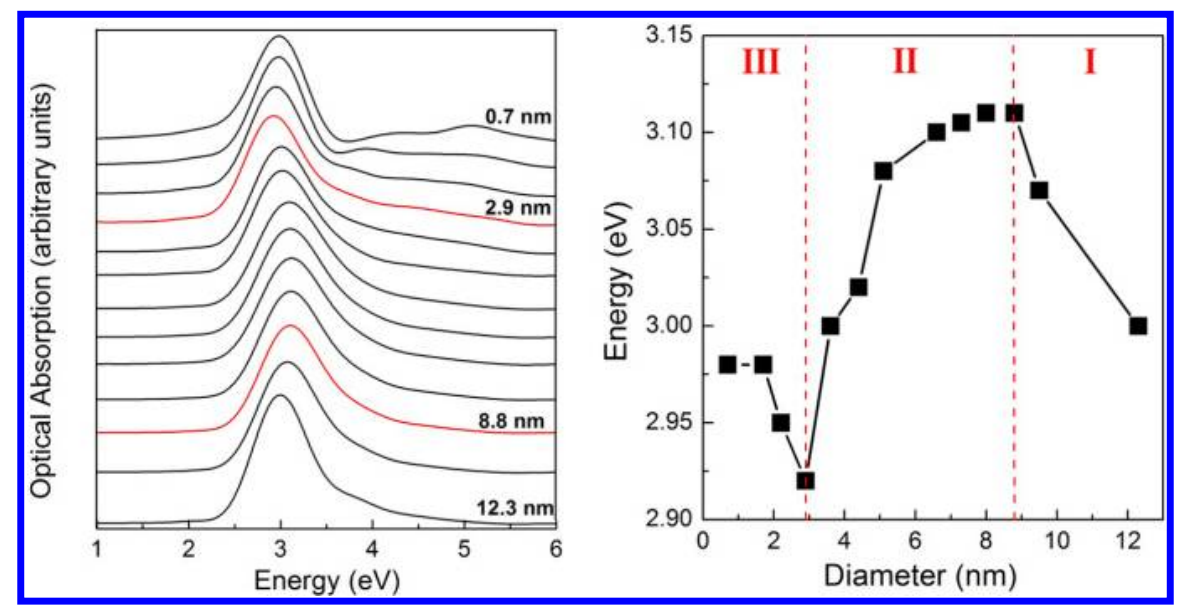

Figure 2. (Left): The optical absorption of Na nanoparticles for $d=12.3,9.5,8.8,8.0,7.3,6.6,5.1,4.4,3.6,2.9,2.2,1.7$, and $0.7 \mathrm{~nm}$. (Right) The peak values (black squares) of the optical absorption spectra as a function of $d$. I, II, and III indicate the three regions of blue shift, red shift, and blue shift of the plasmon resonance energy, respectively.

oscillation across the entire particle in the direction of polarization (negative charge on the left surface and positive charge on the right surface). ${ }^{36}$ The charge density distribution (Friedel oscillations) inside of the particle results from the screening of the charge oscillation on the surface. The charge density distribution at $\omega 2$ can be identified as a Bennet multipole surface plasmon, ${ }^{37,38}$ which originates from electron spill-out at the edge of the nanoparticle. The charge distribution in the polarization direction displays a predominant dipole shape, characterized by two dipoles across the surface. In this case, the surface dipoles are in the same direction. The charge density at $\omega 3$ is similar to that at $\omega 2$ but with two opposite surface dipoles, resembling a breathing mode on the surface. Provided that the particle size is not too large, the two surface dipoles could interact with each other. This interaction gives rise to different energies for the two resonance modes. The $\omega 2$ mode has a lower energy, resulting from a symmetric combination of the surface dipoles (in the same direction), while $\omega 3$ has a higher energy from the antisymmetric combination of the surface dipoles (in the opposite direction). ${ }^{39,40}$

Three competing physical mechanisms underlie the size dependence of LSPRs, including electrodynamic phase retardation, electron spill-out, and quantum size effect. The phase retardation is a classical effect and plays an important role only in larger particles $(d>8 \mathrm{~nm}) .{ }^{41}$ Electron spill-out has a significant impact on the plasmon energy when particles become smaller $(d<5 \mathrm{~nm}){ }^{41}$ The quantum size effect, on the other hand, is prominent only for very small particles $(d<3$ $\mathrm{nm}){ }^{42}$ In region $\mathrm{I}(d>8.8 \mathrm{~nm})$, the phase retardation is at play, and the absorption spectrum is blue-shifted as $d$ decreases from 12.3 to $8.8 \mathrm{~nm}$. This blue shift is well-known both experimentally and computationally from classical theories. ${ }^{15,16}$ The fact that the present TD-OFDFT simulations reproduce this blue shift is a validation of the method as applied to large nanoparticles. In addition, we also find a reasonable quantitative agreement between the classical Mie theory and the present TD-OFDFT for large particles. For example, for $d=10$ and 12 $\mathrm{nm}$, the resonance energies from the Mie theory are 3.20 and $3.17 \mathrm{eV}$, respectively, ${ }^{41}$ close to our corresponding TD-OFDFT values of 3.05 and $3.0 \mathrm{eV}$. In region II $(2.9<d<8.8 \mathrm{~nm})$, the electron spill-out effect becomes more important, which is corroborated by the fact that the relative contribution (the relative height of $\omega 2$ peak among the three plasmon modes) of the spill-out mode $\omega 2$ in $\mathrm{Na}_{1415}{ }^{+}$is greater than that in $\mathrm{Na}_{8217}{ }^{+}$ and $\mathrm{Na}_{55}{ }^{+}$, as shown in Figure 3a. The spill-out of electrons leads to a reduced average electron density; thus, the surface plasmon energy is lower. ${ }^{41}$ In this region, as the particle size decreases, the electron spill-out effect becomes more prominent, and hence, a strong red shift is observed in Figure $2 \mathrm{~b}$. In region III $(d<2.9 \mathrm{~nm})$, the quantum size effect is dominating for all three plasmon modes as the particle becomes increasingly smaller. ${ }^{42}$ The quantum confinement increases the energy-level separations and the excitation energies of surface plasmons, leading to a blue shift with decreasing particle sizes.

For smaller nanoparticles, shape or symmetry has a greater influence on the plasmon resonances. ${ }^{33}$ This may be one of the reasons why there were contradictory experimental results for smaller $\mathrm{Na}$ particles $(N<300){ }^{12-14}$ By examining $\mathrm{Na}$ nanoparticles in the same shape, we can identify the relevant mechanism (quantum size effect) underlying the blue shift size dependence in the smallest nanoparticles. For larger $\mathrm{Na}$ particles $(d>3 \mathrm{~nm})$, there is no experimental result on the plasmonic size dependence. However, there is a recent experiment on Ag nanoparticles stabilized with organic ligands that reported a blue shift as the particle diameter was reduced from $20 \mathrm{~nm}$ then a strong red shift beyond $12 \mathrm{~nm},{ }^{15}$ consistent with our prediction for Na particles. Finally, we note that recent experiments ${ }^{16}$ combining the aberration-corrected transmission electron microscope (TEM) imaging and monochromated scanning TEM electron energy loss spectroscopy may be able to confirm the TD-OFDFT predictions reported in this Letter.

In summary, we have developed a first-principles TDOFDFT method that captures the dynamic response of electrons and the ionic potential accurately. The method offers a comparable accuracy as TD-KSDFT but with much a lower computational cost, thus bridging the gap between classical electromagnetic theory and quantum mechanical theory for plasmonics. The TD-OFDFT method is validated for small $\mathrm{Na}$ particles by comparing its predictions to those from TDKSDFT and experiments. Using the TD-OFDFT method, we study the size dependence of LSPRs for Na nanoparticles in the size range of $12.3>d>0.7 \mathrm{~nm}$. The absorption spectra exhibit a nonmonotonic behavior, a blue shift for $d>8.8 \mathrm{~nm}$, a red shift for $2.9<d<8.8 \mathrm{~nm}$, and a blue shift for $d<2.9 \mathrm{~nm}$, as the particle size decreases. Three principal modes underlying 


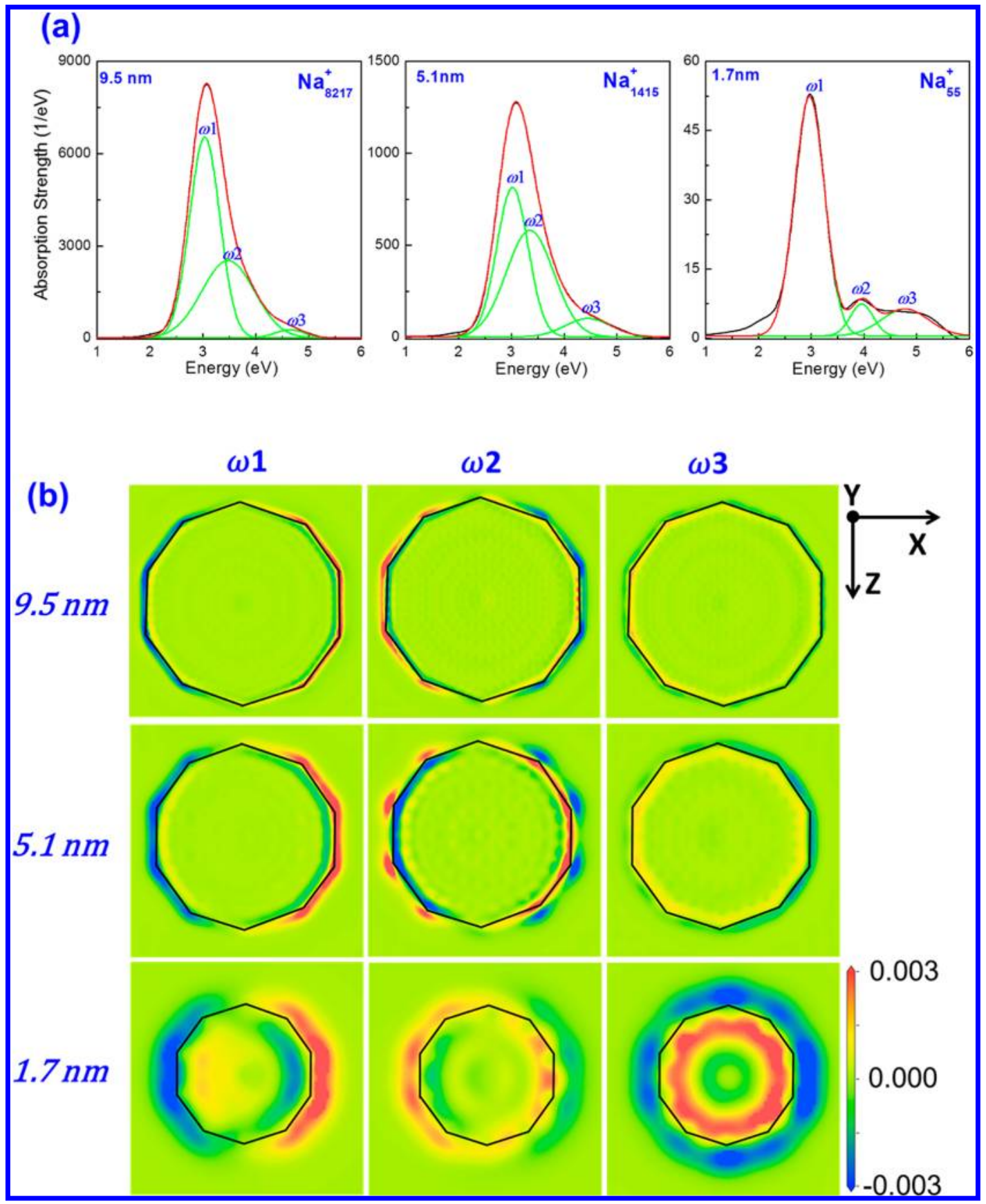

Figure 3. (a) Optical absorption spectrum (black curve) of Na particles with a diameter of $9.5\left(\mathrm{Na}_{8217}{ }^{+}\right), 5.1\left(\mathrm{Na}_{1415}{ }^{+}\right)$, and $1.7 \mathrm{~nm}\left(\mathrm{Na}_{55}{ }^{+}\right)$. The three resolved Gaussian spectra are shown in green centered at $\omega 1, \omega 2$, and $\omega 3$, respectively. The sum of the three Gaussians is shown in red, matching well the original spectrum (black curve). (b) The induced charge density (in atomic unit) evaluated at the frequency $\omega 1, \omega 2$, and $\omega 3$ in response to a horizontal polarization (along $x$ axis). The magnitude of the charge density is color-coded.

plasmonic responses are identified, and their physical origins are elucidated. Three competing mechanisms, phase retardation, electron spill-out, and quantum confinement, are responsible for the nonmonotonic size dependence. The present study resolves the long-standing experimental controversies and sheds light on the size-dependent LSPRs in metallic nanoparticles. Finally, because solvent effects are important for plasmonic applications, one may extend the TD-OFDFT method to incorporate the solvent effects approximately by using an electrostatic continuum solvent model. $^{43}$

\section{COMPUTATIONAL DETAILS}

TD-OFDFT and TD-KSDFT calculations of Na nanoparticles were performed using a code developed in our group and the Octopus code, ${ }^{44}$ respectively. For both codes, real space grids were used to propagate the Kohn-Sham orbitals (TD-KSDFT) and the charge density (TD-OFDFT) in real time under the influence of time-varying electromagnetic fields. A local density approximation $^{45}$ for the electron exchange and correlation was used in both the ground-state and excited-state calculations. The ionic potential of $\mathrm{Na}$ was described by a local pseudopotential $^{27}$ and the Troullier-Martins pseudopotential ${ }^{46}$ in the TD-OFDFT and TD-KSDFT calculations, respectively. 
In the TD-OFDFT calculations, we have performed a convergence check on the size of the supercell and found that the dimension of the cubic supercell (2.5 times of the diameter of the $\mathrm{Na}$ nanoparticle) was sufficient to eliminate particle-particle interactions. The $\mathrm{Na}$ nanoparticle was placed at the center of the supercell. A uniform mesh grid with a spacing of $0.35 \AA$ was used over which the charge density and potential were calculated in both TD-OFDFT and TD-KSDFT. The simulation zone in TD-OFDFT, beyond which the charge density vanishes, was defined by assigning a sphere with a radius of $8 \AA$ around each atom. This choice of radius is necessary for numerical convergence. If electrons are placed too far from the atoms, it becomes exceedingly difficult to converge the results. Fast Fourier transform was used to efficiently calculate the Coulomb potential and the convolution integrals in the kinetic energy functional. We examined $13 \mathrm{Na}$ nanoparticles containing 17885, 8217, 6525, 5083, 3871, $2869,1415,923,561,309,147,55$, and 13 atoms, with corresponding diameters ranging from 12.3 to $0.7 \mathrm{~nm}$. The linear response calculations were performed by propagating the electron wave packets under perturbation of an impulse field $E(t)=E_{\text {kick }} \delta(t)$ for both the TD-OFDFT and TD-KSDFT calculations. ${ }^{47}$ In the real time propagation, the electronic wave packets were evolved for 7500 steps with a time step of $\Delta t=$ $0.0015 \hbar / \mathrm{eV}$.

The geometries of the Na particles with diameter $d \geq 1.7 \mathrm{~nm}$ (55 atoms) were relaxed using the OFDFT method. For the smallest particles, $\mathrm{Na}_{5}{ }^{+}, \mathrm{Na}_{7}{ }^{+}, \mathrm{Na}_{9}{ }^{+}$, and $\mathrm{Na}_{13}{ }^{+}$, the geometries were optimized using KSDFT.

\section{ASSOCIATED CONTENT}

\section{S Supporting Information}

Constructing local pseudopotential of $\mathrm{Na}$. Figure 1: the $\mathrm{Na}$ bulk local pseudopotential in the real space. Fitting dynamic kinetic energy potential (DKEP) parameters. Table 1: a set of parameters used to fit the DKEP. Figure 2: the dynamical susceptibilities as a function of wave vector $q$. Validation of the TD-OFDFT method as compared to experiments and TDKSDFT. Figure 3: the optical absorption of $\mathrm{Na}_{5}{ }^{+}, \mathrm{Na}_{7}{ }^{+}$, and $\mathrm{Na}_{55}{ }^{+}$particles from TD-OFDFT and TD-KSDFT calculations and experiments. This material is available free of charge via the Internet at http://pubs.acs.org.

\section{AUTHOR INFORMATION}

\section{Corresponding Author}

*E-mail: ganglu@csun.edu.

\section{Notes}

The authors declare no competing financial interest.

\section{ACKNOWLEDGMENTS}

We gratefully acknowledge discussions with Chen Huang, Yi Gao, Isaac Shin, and Junchao Xia. The work was supported by the Army Research Office (W911NF-12-1-0072) and NSF (CHE-1112500).

\section{REFERENCES}

(1) Kelly, K. L.; Coronado, E.; Zhao, L. L.; Schatz, G. C. The Optical Properties of Metal Nanoparticles: The Influence of Size, Shape, and Dielectric Environment. J. Phys. Chem. B 2003, 107, 668-677.

(2) Jin, R.; Cao, Y. C.; Hao, E.; Métraux, G. S.; Schatz, G. C.; Mirkin, C. A. Controlling Anisotropic Nanoparticle Growth through Plasmon Excitation. Nature 2003, 425, 487-490.
(3) Lee, K.-S.; El-Sayed, M. A. Gold and Silver Nanoparticles in Sensing and Imaging: Sensitivity of Plasmon Response to Size, Shape, and Metal Composition. J. Phys. Chem. B 2006, 110, 19220-19225.

(4) Mock, J. J.; Barbic, M.; Smith, D. R.; Schultz, D. A.; Schultz, S. Shape Effects in Plasmon Resonance of Individual Colloidal Silver Nanoparticles. J. Chem. Phys. 2002, 116, 6755-6759.

(5) Munechika, K.; Smith, J. M.; Chen, Y.; Ginger, D. S. Plasmon Line Widths of Single Silver Nanoprisms as a Function of Particle Size and Plasmon Peak Position. J. Phys. Chem. C 2007, 111, 18906-18911.

(6) Nagpal, P.; Lindquist, N. C.; Oh, S.-H.; Norris, D. J. Ultrasmooth Patterned Metals for Plasmonics and Metamaterials. Science 2009, 325, 594-597.

(7) Banholzer, M. J.; Millstone, J. E.; Qin, L.; Mirkin, C. A. Rationally Designed Nanostructures for Surface Enhanced Raman Spectroscopy. Chem. Soc. Rev. 2008, 37, 885-897.

(8) Lal, S.; Link, S.; Halas, N. J. Nano-Optics from Sensing to Waveguiding. Nat. Photonics 2007, 1, 641-648.

(9) Fang, N.; Lee, H.; Sun, C.; Zhang, X. Sub-Diffraction-Limited Optical Imaging with a Silver Superlens. Science 2005, 308, 534-537.

(10) Yavuz, M. S.; Chen, Y. Y.; Chen, J. Y.; Cobley, C. M.; Zhang, Q.; Rycenga, M.; Xie, J. W.; Kim, C.; Song, K. H.; Schwartz, A. G.; et al. Gold Nanocages Covered by Smart Polymers for Controlled Release with Near-Infrared Light. Nat. Mater. 2009, 8, 935-939.

(11) Lal, S.; Clare, S. E.; Halas, N. J. Nanoshell-Enabled Photothermal Cancer Therapy: Impending Clinical Impact. Acc. Chem. Res. 2008, 41, 1842-1851.

(12) Parks, J. H.; McDonald, S. A. Evolution of the Collective-Mode Resonance in Small Adsorbed Sodium Clusters. Phys. Rev. Lett. 1989, 62, 2301-2304.

(13) Reiners, T.; Ellert, C.; Schmidt, M.; Haberland, H. SizeDependence of the Optical Response of Spherical Sodium Clusters. Phys. Rev. Lett. 1995, 74, 1558-1561.

(14) Chris Wang, C. R.; Pollack, S.; Dahlseid, T. A.; Koretscky, G. M.; Kappes, M. M. Photodepletion Probes of $\mathrm{Na}_{5}, \mathrm{Na}_{6}$, and $\mathrm{Na}_{7}$. Molecular Dimensionality Transition (2D-3D)? J. Chem. Phys. 1992, 96, 7931-7937.

(15) Peng, S.; McMahon, J. M.; Schatz, G. C.; Gray, S. K.; Sun, Y. Reversing the Size-Dependence of Surface Plasmon Resonances. Proc. Natl. Acad. Sci. U.S.A. 2010, 107, 14530-14534.

(16) Scholl, J. A.; Koh, A. L.; Dionne, J. A. Quantum Plasmon Resonances of Individual Metallic Nanoparticles. Nature 2012, 483, 421-427.

(17) Morton, S. M.; Silverstein, D. W.; Jensen, L. Theoretical Studies of Plasmonics Using Electronic Structure Methods. Chem. Rev. 2011 , $111,3962-3994$.

(18) Link, S.; El-Sayed, M. A. Optical Properties and Ultrafast Dynamics of Metallic Nanocrystals. Annu. Rev. Phys. Chem. 2003, 54, 331-366.

(19) Marinica, D. C.; Kazansky, A. K.; Pordlander, P.; Aizpurua, J.; Borisov, A. G. Quantum Plasmonics: Nonlinear Effects in the Field Enhancement of a Plasmonic Nanoparticle Dimer. Nano Lett. 2012, $12,1333-1339$.

(20) Zuloaga, J.; Prodan, E.; Nordlander, P. Quantum Description of the Plasmon Resonances of a Nanoparticle Dimer. Nano Lett. 2009, 9, $887-891$.

(21) Teperik, T. V.; Nordlander, P.; Aizpurua, J.; Borisov, A. G. Robust Subnanometric Plasmon Ruler by Rescaling of the Nonlocal Optical Response. Phys. Rev. Lett. 2013, 110, 263901.

(22) Teperik, T. V.; Nordlander, P.; Aizpurua, J.; Borisov, A. G. Quantum Effects and Nonlocality in Strongly Coupled Plasmonic Nanowire Dimers. Opt. Express 2013, 21, 27306-27325.

(23) Cottancin, E.; Celep, G.; Lermé, J.; Pellarin, M.; Huntzinger, J. R.; Vialle, J. L.; Broyer, M. Optical Properties of Noble Metal Clusters as a Function of the Size: Comparison between Experiments and a Semi-Quantal Theory. Theor. Chem. Acc. 2006, 116, 514-523.

(24) Prodan, E.; Nordlander, P. Structural Tunability of the Plasmon Resonances in Metallic Nanoshells. Nano Lett. 2003, 3, 543-547. 
(25) Domps, A.; Reinhard, P. G.; Suraud, E. Time-Dependent Thomas-Fermi Approach for Electron Dynamics in Metal Clusters. Phys. Rev. Lett. 1998, 80, 5520-5523.

(26) Neuhauser, D.; Pistinner, S.; Coomar, A.; Zhang, X.; Lu, G. Dynamic Kinetic Energy Potential for Orbital-Free Density Functional Theory. J. Chem. Phys. 2011, 134, 144101.

(27) Huang, C.; Carter, E. A. Transferable Local Pseudopotentials for Magnesium, Aluminum and Silicon. Phys. Chem. Chem. Phys. 2008, 10, 7109-7120.

(28) Gonze, X.; Beuken, J.-M.; Caracas, R.; Detraux, F.; Fuchs, M.; Rignanese, G.-M.; Sindic, L.; Verstraete, M.; Zerah, G.; Jollet, F.; et al. First-Principles Computation of Material Properties: The ABINIT Software Project. Comput. Mater. Sci. 2002, 25, 478-492.

(29) Wang, Y. A.; Govind, N.; Carter, E. A. Orbital-Free KineticEnergy Density Functionals with a Density-Dependent Kernel. Phys. Rev. B 1999, 60, 16350-16358. Erratum: Phys. Rev. B 2001, 64, 089903.

(30) Ke, Y.; Libisch, F.; Xia, J.; Wang, L. W.; Carter, E. A. AngularMomentum-Dependent Orbital-Free Density Functional Theory. Phys. Rev. Lett. 2013, 111, 066402.

(31) Bonačić-Koutecký, V.; Pittner, J.; Fuchs, C.; Fantucci, P.; Guest, M. F.; Koutecký, J. Ab Initio Predictions of Structural and Optical Response Properties of $\mathrm{Na}_{n}^{+}$Clusters: Interpretation of Depletion Spectra at Low Temperature. J. Chem. Phys. 1996, 104, 1427-1440.

(32) Schmidt, M.; Ellert, C.; Kronmüller, W.; Haberland, H. Temperature Dependence of the Optical Response of Sodium Cluster Ions $\mathrm{Na}_{\mathrm{n}}{ }^{+}$, with $4 \leq \mathrm{n} \leq 16$. Phys. Rev. B 1999, 59, 10970-109709.

(33) Yin, H. F.; Zhang, H. Collectivity of Plasmon Excitations in Small Sodium Clusters with Planar Structure. Physica B 2012, 407, 416-420.

(34) Li, J. H.; Hayashi, M.; Guo, G. Y. Plasmonic Excitations in Quantum-Sized Sodium Nanoparticles Studied by Time-Dependent Density Functional Calculations. Phys. Rev. B 2013, 88, 155437.

(35) Diaz-Egea, C.; Sigle, W.; Van Aken, P. A.; Molina, S. I. High Spatial Resolution Mapping of Surface Plasmon Resonance Modes in Single and Aggregated Gold Nanoparticles Assembled on DNA Strands. Nanosci. Res. Lett. 2013, 8, 337.

(36) Myroshnychenko, V.; Rodríguez-Fernández, J.; Pastoriza-Santos, I.; Funston, A. M.; Novo, C.; Mulvaney, P.; Liz-Marzán, L. M.; García de Abajo, F. J. Modelling the Optical Response of Gold Nanoparticles. Chem. Soc. Rev. 2008, 37, 1792-1805.

(37) Stella, L.; Zhang, P.; García-Vidal, F. J.; Rubio, A.; GarcíaGonzález, P. Performance of Nonlocal Optics when Applied to Plasmonic Nanostructures. J. Phys. Chem. C 2013, 117, 8941-8949.

(38) Bennet, A. J. Influence of the Electron Charge Distribution on Surface-Plasmon Dispersion. Phys. Rev. B 1970, 1, 203-207.

(39) Prodan, E.; Radloff, C.; Halas, N. J.; Nordlander, P. A Hybridization Model for the Plasmon Response of Complex Nanostructures. Science 2003, 302, 419-422.

(40) Nordlander, P.; Oubre, C.; Prodan, E.; Li, K.; Stockman, M. I. Plasmon Hybridization in Nanoparticle Dimers. Nano Lett. 2004, 4, 899-903.

(41) Kreibig, U.; Vollmer, M. Optical Properties of Metal Clusters; Springer: Berlin, Germany, 1995.

(42) Zhu, M.; Aikens, C. M.; Hollander, F. J.; Schatz, G. C.; Jin, R. Correlating the Crystal Structure of a Thiol-Protected $\mathrm{Au}_{25}$ Cluster and Optical Properties. J. Am. Chem. Soc. 2008, 130, 5883-5885.

(43) Fattebert, J. L.; Gygi, F. Density Functional Theory for Efficient $\mathrm{Ab}$ Initio Molecular Dynamics Simulations in Solution. J. Comput. Chem. 2002, 23, 662-666.

(44) Marques, M. A. L.; Castro, A.; Bertsch, G. F.; Rubio, A. Octopus: A First-Principles Tool for Excited Electron-Ion Dynamics. Comput. Phys. Commun. 2003, 151, 60-78.

(45) Ceperley, D. M.; Alder, B. J. Ground State of the Electron Gas by a Stochastic Method. Phys. Rev. Lett. 1980, 45, 566-569.

(46) Troullier, N.; Martins, J. L. Efficient Pseudopotentials for PlaneWave Calculations. Phys. Rev. B 1991, 43, 1993-2006.

(47) Yabana, K.; Bertsch, G. F. Time-Dependent Local-Density Approximation in Real Time. Phys. Rev. B 1996, 54, 4484-4487. 